\title{
Protective Effect of Ginsenoside Against Acute Renal Failure via Reduction of Renal Oxidative Stress and Enhanced Expression of ChAT in the Proximal Convoluted Tubule and ERK1/2 in the Paraventricular Nuclei
}

\author{
J. ZHOU ${ }^{1,2^{*}}$, H. A. ZHANG ${ }^{1,3^{*}}$, Y. LIN $^{4}$, H. M. LIU ${ }^{5}$, Y. M. CUI ${ }^{5}$, Y. XU ${ }^{5}$, N. ZHAO ${ }^{5}$, \\ J. M. MA ${ }^{6}$, K. FAN ${ }^{6}$, C. L. JIANG ${ }^{1}$ \\ ${ }^{*}$ These authors contributed equally to this work.
}

${ }^{1}$ Department of Physiology, Dalian Medical University, Dalian, China, ${ }^{2}$ Department of Dermatology, Dalian Economic \& Technological Developmental Area Hospital, Dalian, China, ${ }^{3}$ Division of Cardiovascular Research, State Key Laboratory of Biomembrane and Membrane Biotechnology, Institute of Zoology, Chinese Academy of Sciences, Beijing, China, ${ }^{4}$ Department of Pharmacology, ${ }^{5}$ Department of Clinical Medicine, ${ }^{6}$ Department of Anatomy, Dalian Medical University, Dalian, China

Received December 15, 2013

Accepted March 7, 2014

On-line June 5, 2014

\section{Summary}

Generation of reactive oxygen species significantly contributes to the pathogenesis of acute renal failure (ARF) induced by myoglobin release. Ginsenosides (GS), the principal active ingredients of ginseng, is considered as an extremely good antioxidative composition of Chinese traditional and herbal drugs. The purpose of the present study was to investigate the protective effect of ginsenoside in rats with ARF on the changes of cholinergic nervous system in the kidney as well as on the involvement of mitogen-activated protein kinases (MAPK) in the hypothalamic paraventricular nuclei (PVN). In our assay, glycerolinduced acute renal failure in rats was employed to study the protective effects of ginsenoside. Our results indicated that the treatment of ARF rats with ginsenosides for $48 \mathrm{~h}$ significantly reduced lipid peroxidation, restored the superoxide dismutase (SOD) level. Meanwhile, the obvious increase of choline acetyltransferase-immunoreactivity (ChAT-IR) in the proximal convoluted tubular cells (PCT) was observed by immunohistochemistry in ARF+GS group. The same effect was also observed in the changes of $p$-ERK1/2-IR in the hypothalamic paraventricular nuclei. Our results suggest that ginsenoside administered orally may have a strong renal protective effect against glycerol-induced ARF, reduce the renal oxidative stress, and ginsenoside can also activate the cholinergic system in PCT, simultaneously MAPK signal pathway in the PVN was also activated.

\section{Key words}

Ginsenoside - Acute renal failure - Proximal convoluted tubular cells $\bullet$ Choline acetyltransferase $\bullet$ Paraventricular nuclei

\section{Corresponding author}

C. L. Jiang, Department of Physiology, Dalian Medical University, Dalian, Liaoning, China. E-mail: chunling_jiang2006@163.com

\section{Introduction}

Almost $10-15 \%$ of all cases of acute renal failure (ARF) may account for traumatic and nontraumatic clinical conditions manifested with muscle fiber disintegration or rhabdomyolysis (Vlahović et al. 2007). There is increasing evidence indicating that oxidative stress plays an important role in the pathogenesis of rhabdomyolysis-induced myoglobinuric acute renal failure (Ustundag et al. 2009). ARF is usually reversible, the kidney being able to recover from almost complete loss of function. Nevertheless, ARF is associated with major inpatient morbidity and mortality, reflecting the severity of the casual illnesses and the high frequency of complications (Yoon et al. 2008). Thus, early and effective renoprotective treatments are of utmost 
importance. In recent years, great effort has been focused on traditional and herbal medicine without toxic effects to provide a novel therapeutic agent for ARF.

Ginsenosides (GS), the unique constituents and secondary metabolites of the Panax species, have been known to be the pharmacologically active ingredients of ginseng and has a wide range of pharmacological and physiological actions due to its antioxidant and free radicals scavenging properties (Attele et al. 1999). It has been reported that Ginsenosides Rh4 significantly reduced the cisplatin-induced nephrotoxicity in cultured renal proximal tubular epithelial cells in a dose-dependent manner (Baek et al. 2006). Similarly, ginsenoside-Rd ameliorated the renal injury induced by cephaloridine through up-regulating the activities of the antioxidation enzymes superoxide dismutase and catalase (Yokozawa and Owada 1999). However, the effect of GS administered orally on ARF has been seldom reported yet and little is known about its cellular and molecular mechanism.

The primary role of the kidney is to maintain the volume and electrolyte composition of body fluids and, in this way, to regulate the blood pressure. In proximal tubular cells, cholinergic agonists have been shown to rapidly increase basolateral $\mathrm{Na}-\mathrm{HCO}_{3}$ cotransport (NBC) activity. And the classic mitogen-activated protein kinase (MAPK) pathway activation is coupled to increase NBC activity (Robey et al. 2001). It suggested that renal cholinergic system participated in regulation of epithelial ion transport and there was an association between MAPK pathway and cholinergic system. Recent studies suggested that acetylcholine prevented the hypoxiainduced apoptosis of mouse embryonic stem (ES) cells by inhibiting the reactive oxygen species (ROS) production. Meanwhile, acetylcholine also prevented angiotensin IIinduced oxidative stress and apoptosis in H9c2 cells (Liu et al. 2011). These findings suggested that cholinergic system play a positive role in oxidative stress. However, whether renal cholinergic system activity is changed in ARF rats involved in oxidative stress is unknown.

The hypothalamic paraventricular nuclei (PVN) play a prominent role in the central regulation of water intake, urinary volume and sodium excretion, maintaining the homeostasis and regulate organismal function (de Arruda Camargo et al. 2010). MAPKs are a family of Ser/Thr protein kinases widely conserved among eukaryotes and are involved in many cellular programs such as cell proliferation, differentiation, survival movement, and cell death. The extracellular signal- regulated kinases (ERK) represent one of the four subfamilies of MAP kinases. It is reported that survival factors inhibit cell death by activating specific signaling pathways, including stimulation of protein-tyrosine phosphorylation and activation of ERK-1/2, which then lead to the inhibition of the apoptotic signaling cascade (Baldanzi et al. 2002). This finding implied activation of MAPK pathways may participate in cytoprotection. Increasing evidences have shown that ACh plays a key role in the regulation of cell morphogenesis, proliferation and differentiation in many species; the growthregulatory action of $\mathrm{ACh}$ is associated with signal transduction pathways involving MAPK signaling pathways (Ma et al. 2000). The paraventricular nuclei plays an important role in the regulation of fear, anxiety and stress (Herring et al. 2004). The neural cell adhesion molecule (NCAM) mimetic peptide C3d promoted ChAT activity in septal neurons through the stimulation of fibroblast growth factor receptor (FGFR) and MAPK cascade, in this way, played a protective role (Burgess et al. 2009). Therefore we hypothesized that MAPK might be involved in the protective role of cholinergic system. Our previous studies showed MAPK signaling pathway was activated in locus ceruleus of ARF rats (Zhang et al. 2010). However, whether hypothalamic MAPK signal pathway was activated in glycerol-induced ARF rats remains unclear.

Based on these findings, it could be speculated that ginsenosides might have protective effects and there might be some interactions between cholinergic system in the kidney and MAPK signal pathway in the hypothalamic paraventricular nuclei in glycerol-induced ARF rats. Therefore, we examined 1) the changes of malondialdehyde (MDA) and superoxide dismutase (SOD) in renal cortex homogenate of ARF rats treated with ginsenoside for $48 \mathrm{~h}, 2$ ) the changes of choline acetyltransferase-immunoreactivity (ChAT-IR) in the PCT of glycerol-induced ARF rats treated with ginsenoside for $48 \mathrm{~h}, 3$ ) the changes of phospho-ERK1/2immunoreactivity (p-ERK1/2-IR) in the hypothalamic paraventricular nuclei of glycerol-induced ARF rats treated with ginsenoside for $48 \mathrm{~h}$.

\section{Methods}

Animals

Healthy male Sprague-Dawley rats (Dalian Medical University Animal Center, China) weighing 180$220 \mathrm{~g}$ were kept on a $12 \mathrm{~h} / 12 \mathrm{~h}$ light/dark cycle and 
received food and water ad libitum at room temperature. During this time, rats were handled daily to avoid stressinduced expression on the day of the experiment. Special care was taken to minimize animal suffering and to reduce the number of animals used to the minimum necessary.

\section{Animals' treatment}

Sixty four male rats were used in this study. After several days of adaptation, they were deprived of water for $16 \mathrm{~h}$ from 5 p.m. to 9 a.m.

Forty rats for the experiment in vivo were divided randomly into four groups( $n=10$ per group): ARF + physiological saline (NS) group, ARF + GS group, NS+NS group and NS+GS group. After deprived of water for $16 \mathrm{~h}, \mathrm{ARF}+\mathrm{NS}$ group and $\mathrm{ARF}+\mathrm{GS}$ group were given intramuscularly with $10 \mathrm{ml} / \mathrm{kg}$ body weight of $50 \%$ (vol/vol) glycerol solution distributed equally in both hind limbs. As soon as the model was established, ARF+GS group was given $25 \mathrm{mg}$ GS in $2 \mathrm{ml}$ NS using a stomach tube, at the same time, ARF+NS group was given $2 \mathrm{ml}$ NS. The treatment of the other two groups were similar to them. They were treated with GS or NS for two consecutive days (once per $6 \mathrm{~h}$, three times per day).

Another 24 rats for immunohistochemistry were also divided into 4 groups and treated as described in the experiment in vivo.

\section{Experiment in vivo}

After treatment with ginsenoside or physiological saline for $48 \mathrm{~h}$, kidney was removed, then renal cortex was homogenized with ice-cold physiological saline. MDA content, a measure of lipid peroxidation, was assayed in the form of thiobarbituric acid reacting substances (TBARS) using commercial reagents (Nanjing Jiancheng Bioengineering Institute, P.R. China). The SOD was measured by the method of xanthine oxidase using commercial reagents (Nanjing Jiancheng Bioengineering Institute, P.R. China). It was observed by measuring the absorbance at $550 \mathrm{~nm}$.

\section{Immunohistochemistry analysis}

Renal sections ( $8 \mu \mathrm{m}$ thick) were cut on Cryostat at $-12{ }^{\circ} \mathrm{C}$ and placed onto low-iron clear glass slides. The sections above were incubated in $30 \% \mathrm{H}_{2} \mathrm{O}_{2}$ in methanol for $10 \mathrm{~min}$ and incubated with $0.4 \%$ pepsin for $1 \mathrm{~h}$ at $37^{\circ} \mathrm{C}$. The sections were washed three times in PBS $10 \mathrm{~min}$ and incubated in the primary antibody (ChAT-Ab, 1:200 Boster Company, P.R. China) $1 \mathrm{~h}$ at room temperature. The sections were then rinsed three times in PBS for $5 \mathrm{~min}$ and incubated in the biotinylated-second antibody (Boster Company, P.R. China) at room temperature for $1 \mathrm{~h}$. Then the sections were again rinsed three times in PBS for $10 \mathrm{~min}$ and incubated with the avidin-biotin complex ABC (Boster Company, P.R. China) at room temperature for $1 \mathrm{~h}$. Diaminobenzidine (DAB, Sigma Co, USA) and hematoxylin were used for signal detection. The control sections were incubated with PBS instead of primary antibody. Image Pro Plus image analysis system was used to analyze ChAT-IR positive granules. Kidney sections were observed in a microscope at $20 \times$ magnification. Optical density of ChAT-IR positive granules was calculated per area and per group.

$50 \mu \mathrm{M}$ thick coronal brain sections were sliced on a vibratome. The identification of paraventricular nucleus (bregma $-1.80 \mathrm{~mm}$ to $-2.12 \mathrm{~mm}$ ) was based on the atlas by Paxinos and Watson. The sections above were rinsed three times in PBS 10 min and then incubated with $0.2 \%$ Triton for $5 \mathrm{~min}$. The sections were rinsed three times in PBS $10 \mathrm{~min}$ and incubated with bovine serum albumin (2 \% BSA) (Sigma Co., USA) for $1 \mathrm{~h}$. Thereafter the sections were incubated in the primary antibody (p-ERK1/2-Ab, 1:100, Boster Company, P.R. China) overnight at $4{ }^{\circ} \mathrm{C}$. The sections were then rinsed three times in PBS for $10 \mathrm{~min}$ and incubated with $2 \%$ BSA for 20 min. Subsequently, the sections were rinsed three times in PBS for $10 \mathrm{~min}$, and further incubated in the biotinylated-second antibody (Boster Company, P.R. China) at room temperature for 2 h. Finally, the sections were again rinsed three times in PBS for $10 \mathrm{~min}$ and incubated with the avidin-biotin complex ABC (Boster Company, P.R. China) at room temperature for $2 \mathrm{~h}$. Diaminobenzidine (DAB, Sigma Co, USA) was used for signal detection. The control sections were incubated with PBS instead of primary antibody. The HPIAS series colorful pathology photographic system was used to analyze p-ERK1/2-IR positive neurons. The brain sections were observed in a $20 \times$ magnification. The number and optical density of p-ERK1/2-IR positive neurons were calculated per area and per group. 

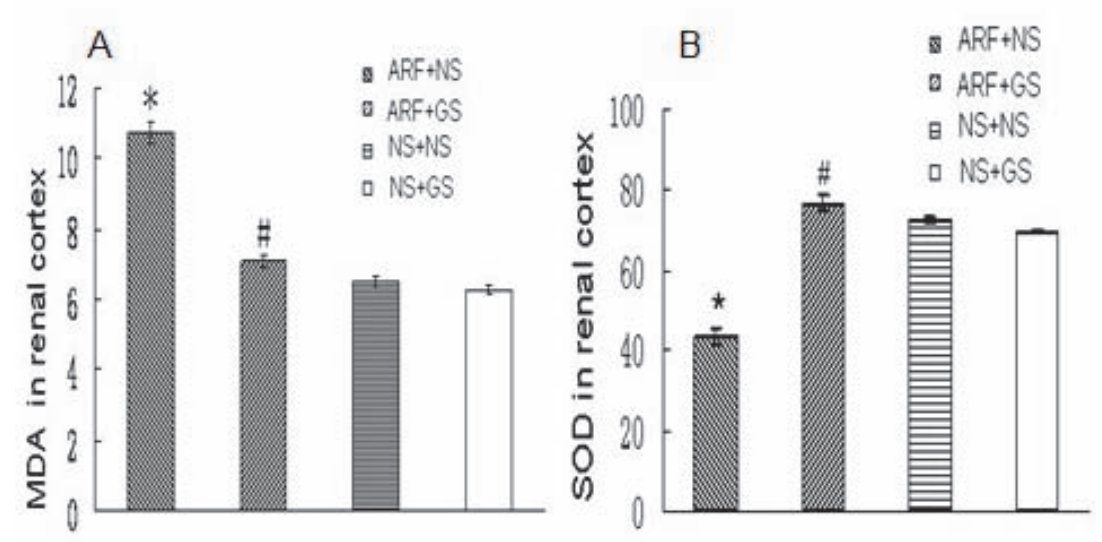

Fig. 1. Changes of MDA and SOD in renal cortex homogenate in the ARF rats treated with GS for $48 \mathrm{~h}$. A. MDA in renal cortex; B. SOD in renal cortex. Data are mean \pm S.E.M, $n=10$ (* $\mathrm{P}<0.05 \mathrm{ARF}+\mathrm{NS}$ group vs. NS+NS group, ${ }^{*} \mathrm{P}<0.05$ ARF+GS group vs. ARF+NS group).

\section{Statistical analysis}

All data were expressed as mean \pm S.E.M. Statistical evaluation was done using ANOVA with post hoc test of LSD in Equal Variances Assumed. In all comparisons, statistical significance was set at $\mathrm{P}<0.05$.

\section{Results}

\section{Effect of GS on renal MDA and SOD level}

Glycerol-treated rats (ARF+NS group) showed a significant lipid peroxidation as indicated by a marked increase of renal MDA level and decreased SOD level as compared to those in NS+NS group. However, treatment with GS for $48 \mathrm{~h}$ (ARF+GS group) significantly reduced the MDA level and restored the SOD level as compared to $\mathrm{ARF}+\mathrm{NS}$ group (Fig. $1, \mathrm{P}<0.05$ ).

Effect of GS on the changes of ChAT-IR in the PCT in ARF rats induced by glycerol

In the NS+NS group, ChAT-IR positive granules were detected very weakly in proximal convoluted tubular cells (Fig. 2C). Optical density of ChAT-IR positive granules in the ARF+NS group was significantly increased in PCT compared with that in the NS+NS group (Fig. 2A, $\mathrm{P}<0.05$ ). There was a further increase of optical density of ChAT-IR positive granules in PCT of $\mathrm{ARF}+\mathrm{GS}$ group (Fig. 2B, $\mathrm{P}<0.05$ ) when compared with that in ARF+NS group. No significant difference was found in optical density of ChAT-IR positive granules in PCT, when the NS +GS group (Fig. $2 \mathrm{D}, \mathrm{P}<0.05$ ) was compared with the NS+NS group. The data on ChAT-IR in the PCT were summarized in Figure 2.

Effect of GS on the changes of p-ERK1/2-IR in the PVN in $A R F$ rats induced by glycerol

Immunohistochemistry also showed an obvious increase of p-ERK 1/2 -IR in the PVN of ARF+NS group
(Fig. 3A, $\mathrm{P}<0.05$ ); but $\mathrm{p}$-ERK-IR $1 / 2$ was further enhanced in $A R F+G S$ group compared with $A R F+N S$ group (Fig. 3B, $\mathrm{P}<0.05$ ).

\section{Discussion}

Intramuscular administration of hypertonic glycerol is the most common used animal model of experimental ARF. The mechanism of this model is rhabdomyolysis induced by hypertonic glycerol (Ustundag et al. 2009). Hypertonic glycerol produced a large amount of myoglobin to leak into circulation. Myoglobin, along with uric acid, can damage the kidney directly by forming casts within the renal tubules leading to their obstruction. This blockage of renal tubules induced necrosis of renal tubular epithelial cell and produced a large amount of free radical. Myoglobin also can lead to intrarenal vasoconstriction and decreased glomerular filtration rate. Finally, all of these factors cause ARF (Aydogdu et al. 2006, Boutaud and Roberts 2011). Our previous paper reported that glycerol administration resulted in a significant increase in BUN and Cre levels as compared to those in NS+NS group, and the kidneys of rats treated with glycerol showed marked histological changes in cortex (Zhang et al. 2010). Therefore, the animal model of experimental ARF was successful.

It was reported that the iron released from the heme group of the myoglobin. Iron induces the formation of high-activity oxygen free radicals that increase oxidative stress and provoke lipid peroxidation and cellular death (Johnson et al. 2010). It was reported that glycerol caused the renal oxidative stress. Reactive oxygen metabolites were proved to be the key mediators of tissue injury in glycerol-induced ARF model (Ustundag et al. 2009). 

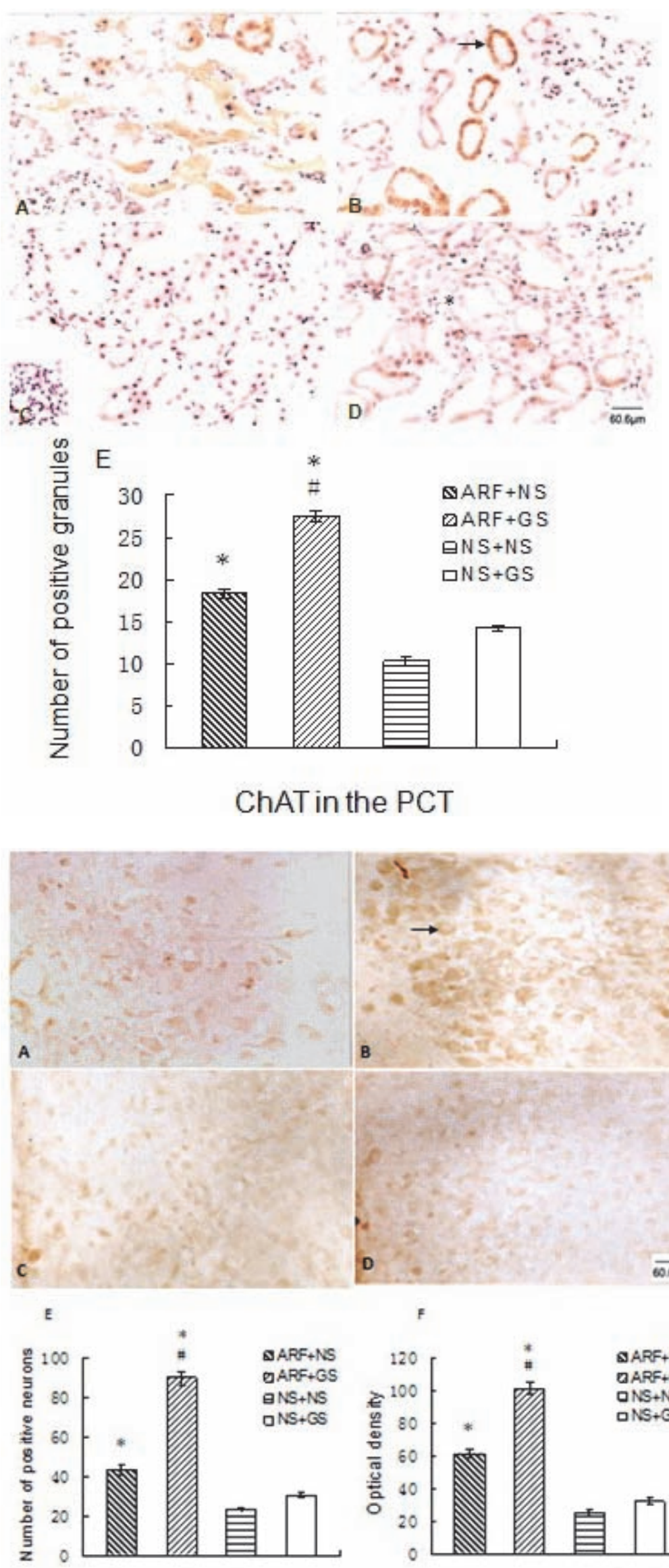

ERK in the PVN
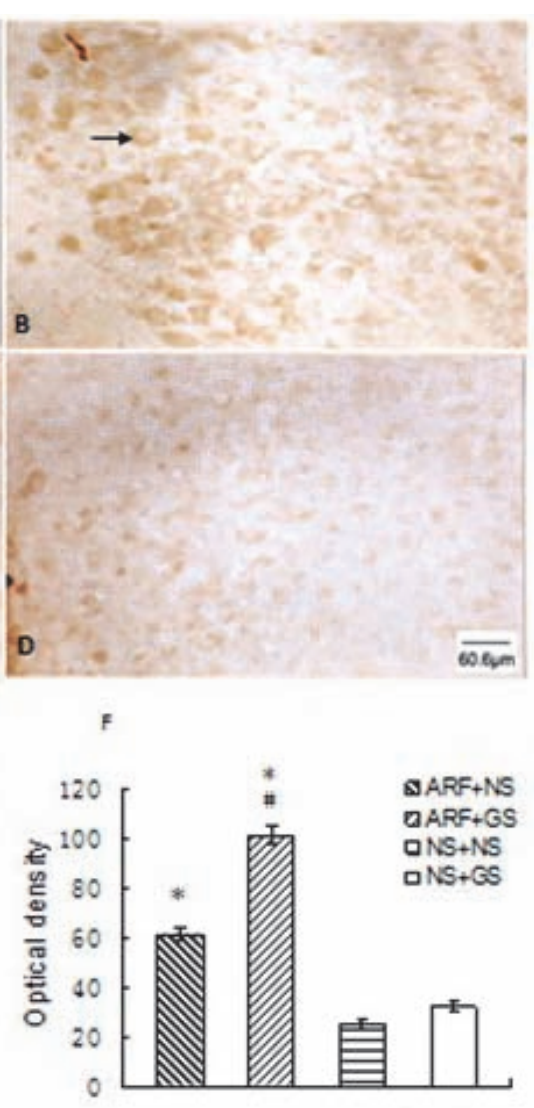

ERK in the PVN

Fig. 3. Changes of $p-E R K 1 / 2-I R$ in $P V N$ in the ARF rats treated with GS for 48 h. A, B, C and $\mathbf{D}$ were stained by $\mathrm{DAB}$. A: ARF+NS group; B: ARF+GS group; C: NS+NS group; D: NS+GS group. Bar indicates $60.6 \mu \mathrm{M}$, arrow points to $p$-ERK1/2-IR positive neurons. $\mathbf{E}$ and $\mathbf{F}$ : quantitative analysis of positive neurons and optical mean density of p-ERK1/2-IR in PVN. * $\mathrm{P}<0.05$ vs. NS+NS group; ${ }^{\#} P<0.05$ vs. ARF+NS group.

Fig. 2. Changes of ChAT-IR in PCT treated with GS for $48 \mathrm{~h}$ in ARF rats. A, B, C and D ARF+GS group; C: NS+NS group; D: NS+GS group. Bar indicates $60.6 \mu \mathrm{M}$, arrow points to ChAT-IR positive granules. E: Quantitative analysis of ChAT-IR positive granules in PCT. ${ }^{*} \mathrm{P}<0.05$ vs. NS+NS group; ${ }^{*} \mathrm{P}<0.05$ vs. ARF+NS group. 
SOD used to scavenge free radicals was decreased when free radicals was increased (Yoon et al. 2008); SOD is a significant index in the research about oxidative damage. In the present study, we observed that MDA level in renal cortex homogenate from ARF rats was significantly increased and the level of SOD in renal cortex homogenate of ARF rats was markedly decreased, indicating that reactive oxidative species were generated in this model in which oxidative damage was enhanced. In addition, we also demonstrated that the level of MDA was significantly decreased and the level of SOD was obviously increased after the treatment with ginsenosides for $48 \mathrm{~h}$. Similarly, ginsenosides exerted a protective effects on the progression of renal damage via inhibiting oxidative stress in type 2 diabetic rats (Kang et al. 2010). Ginsenoside Rb2 through transcription factor AP2 binding sites and its induction greatly activated the SOD gene, upregulated expression of SOD and enhanced antioxidant activity (Kim et al. 1996). Taken together, we supposed ginsenosides, as inhibitors of lipid peroxidation resulting from oxidative stress and as an oxidative free radical scavenger through the increase of SOD, played a crucial role in the protection against the damage associated with rhabdomyolysis.

Previous study have indicated in the dog renal cortex the existence of high-affinity choline uptake and the presence of ChAT, the enzyme that converts choline to acetylcholine and acetylcholine grains were also found in dog renal cortex (Pirola et al. 1991). It was reported that hypoxia increased the intracellular level of reactive oxygen species and induced cell apoptosis in mouse ES cells; but this effect can be inhibited by ACh administration (Kim et al. 2008). It suggested that acetylcholine prevented the hypoxia-induced apoptosis of mouse ES cells by inhibiting the reactive oxygen species production. These evidences implied renal cholinergic system played an important role during oxidative stress. In the present study, we observed that the ChAT-IR was markedly increased in PCT cell of ARF rats, it may be a compensatory mechanism of cholinergic system against the increase of oxyradical and renal ischemia, hypoxia. In addition, we also observed a further increase of the ChAT-IR in the PCT of ARF+GS group, compared with that in ARF+NS groups. Our results suggested that ginsenoside further up-regulated renal cholinergic system activity.

Immunostaining for ERK was intense in various brain regions such as neocortex, hippocampus, hypothalamus, brainstem (Flood et al. 1998). It was reported that several physiologically stressful stimuli, including seizure induction, ischemic insult and electroconvulsive shock, could activate MAPK signal in various brain regions (Imbe et al. 2004). Meanwhile, the MAPK pathway was activated in the process of Ang IIinduced ROS production (Palomeque et al. 2009). NAinduced p38 MAPK activation, through alphaadrenoceptor, had a protective role in cardiomyocytes to antagonize NA-induced cell death (Tsang and Rabkin 2009). It prompted that MAPK signaling pathways activation had a cytoprotective action. Other investigators have reported that the ERK pathway activation was an important step in the regulation of oxidative stressresponse genes by arecoline (Thangjam and Kondaiah 2009). The nature of the signal transduction suggested that activation of ERK1/2 may serve as a convergence site for mAChR activation in COS-7 cells (Rosenblum et al. 2000). It implied that the MAPK signaling pathways activation may influence cholinergic nervous system. A parasite-derived neurotrophic factor stimulated mRNA and protein expression of ChAT in PC12 cell, and activating MAPK signaling pathways is necessary for this effect (Akpan et al. 2008). The neural cell adhesion molecule mimetic peptide C3d promoted ChAT activity in septal neurons through the stimulation of fibroblast growth factor receptor and the activation of the MAPK signaling pathway (Burgess et al. 2009). Therefore, we can speculate that the MAPK signaling pathway was involved in the regulation of ChAT activity.

The results of the present study also showed that of the p-ERK1/2-IR in PVN of ARF rats was significantly increased. It indicates that MAPK signal pathway in the PVN was excited after intramuscular injection of glycerol and might be involved in the regulation of cholinergic system in proximal convoluted tubule. In addition, we also observed that glycerolinduced ARF rats treated with ginsenosides for $48 \mathrm{~h}$ enhanced the p-ERK1/2-IR as well as the ChAT-IR in PCT.

On the basis of the above results, we propose that glycerol-induced ARF causes the changes not only in the kidney such as acute tubular necrosis, the increase of oxidative stress and the upregulation of the ChAT-IR in the PCT, but also in central nervous system such as the upregulation of the p-ERK1/2-IR in the hypothalamic PVN. We suggest that both changes in the PCT and PVN should be one of the compensatory mechanisms both in the kidney and in central nervous system in ARF rats. Our study also shows that oral administration of 
ginsenosides not only further increase renal antioxidative activity by some humoral mechanism, such as activating cholinergic system activity in the PCT, but also activate hypothalamic PVN MAPK signal pathway through some unknown pathways. The possible relationship between the activation of cholinergic system in the PCT and the activation of hypothalamic PVN MAPK signal pathway, and also its correlation with renal protective effect of ginsenosides against ARF will be a focal point in our lab for the future.

\section{Conflict of Interest}

There is no conflict of interest.

\section{Acknowledgements}

This work was supported by grant No. 9910500711 from the Natural Science Foundation of Liaoning Province, China. The present address of Hong-mei Liu: Tangshan Gongren Hospital, Tangshan, Hebei, China.

\section{References}

AKPAN N, CARADONNA K, CHUENKOVA MV, PEREIRAPERRIN M: Chagas' disease parasite-derived neurotrophic factor activates cholinergic gene expression in neuronal PC12 cells. Brain Res 1217: 195-202, 2008.

ATTELE AS, WU JA, YUAN CS: Ginseng pharmacology: multiple constituents and multiple actions. Biochem Pharmacol 58: 1685-1693, 1999.

AYDODGU N, ATMACA G, YALCIN O, TASKIRAN R, TASTEKIN E, KAYMAK K: Protective effects of Lcarnitine on myoglobinuric acute renal failure in rats. Clin Exp Pharmacol Physiol 33: 119-124, 2006.

BAEK SH, PIAO XL, LEE UJ, KIM HY, PARK JH: Reduction of cisplatin-induced nephrotoxicity by ginsenosides isolated from processed ginseng in cultured renal tubular cells. Biol Pharm Bull 29: 2051-2055, 2006.

BALDANZI G, FILIGHEDDU N, CUTRUPI S, CATAPANO F, BONISSONI S, FUBINI A, MALAN D, BAJ G, GRANATA R, BROGLIO F, PAPOTTI M, SURICO N, BUSSOLINO F, ISGAARD J, DEGHENGHI R, SINIGAGLIA F, PRAT M, MUCCIOLI G, GHIGO E, GRAZIANI A: Ghrelin and des-acyl ghrelin inhibit cell death in cardiomyocytes and endothelial cells through ERK1/2 and PI 3-kinase/AKT. J Cell Biol 159: 10291037, 2002.

BOUTAUD O, ROBERTS LJ: Mechanism-based therapeutic approaches to rhabdomyolysis-induced renal failure. Free Radic Biol Med 51: 1062-1067, 2011.

BURGESS A, SAINI S, WENG YQ, AUBERT I: Stimulation of choline acetyltransferase by C3d, a neural cell adhesion molecule ligand. J Neurosci Res 87: 609-616, 2009.

DE ARRUDA CAMARGO GM, DE ARRUDA CAMARGO LA, SAAD WA: On a possible dual role for the lateral septal area 5-HT(1A) receptor system in the regulation of water intake and urinary excretion. Behav Brain Res 215: 122-128, 2010.

FLOOD DG, FINN JP, WALTON KM, DIONNE CA, CONTRERAS PC, MILLER MS, BHAT RV: Immunolocalization of the mitogen-activated protein kinases $\mathrm{p} 42 \mathrm{MAPK}$ and JNK1, and their regulatory kinases MEK1 and MEK4, in adult rat central nervous system. J Comp Neurol 398: 373-392, 1998.

HERRING BE, MAYFIELD RD, CAMP MC, ALCANTARA AA: Ethanol-induced Fos immunoreactivity in the extended amygdala and hypothalamus of the rat brain: focus on cholinergic interneurons of the nucleus accumbens. Alcohol Clin Exp Res 28: 588-597, 2004.

IMBE H, MURAKAMI S, OKAMOTO K, IWAI-LIAO Y, SENBA E: The effects of acute and chronic restraint stress on activation of ERK in the rostral ventromedial medulla and locus coeruleus. Pain 112: 361-371, 2004.

JOHNSON AC, BECKER K, ZAGER RA: Parenteral iron formulations differentially affect MCP-1, HO-1, and NGAL gene expression and renal responses to injury. Am J Physiol 299: F426-F435, 2010.

KANG KS, YAMABE N, KIM HY, PARK JH, YOKOZAWA T: Effects of heat-processed ginseng and its active component ginsenoside 20(S)-Rg3 on the progression of renal damage and dysfunction in type 2 diabetic Otsuka Long-Evans Tokushima Fatty rat. Biol Pharm Bull 33: 1077-1081, 2010. 
KIM MH, KIM MO, HEO JS, KIM JS, HAN HJ: Acetylcholine inhibits long-term hypoxia-induced apoptosis by suppressing the oxidative stress-mediated MAPKs activation as well as regulation of Bcl-2, c-IAPs, and caspase-3 in mouse embryonic stem cells. Apoptosis 13: 295-304, 2008.

KIM YH, PARK KH, RHO HM: Transcriptional activation of the $\mathrm{Cu}$, Zn-superoxide dismutase gene through the AP2 site by ginsenoside Rb2 extracted from a medicinal plant, Panax ginseng. J Biol Chem 271: 24539-24543, 1996.

LIU JJ, LI DL, ZHOU J, SUN L, ZHAO M, KONG SS, WANG YH, YU XJ, ZHOU J, ZANG WJ: Acetylcholine prevents angiotensin II-induced oxidative stress and apoptosis in H9c2 cells. Apoptosis 16: 94-103, 2011.

MA W, MARIC D, LI BS, HU Q, ANDREADIS JD, GRANT GM, LIU QY, SHAFFER KM, CHANG YH, ZHANG L, PANCRAZIO JJ, PANT HC, STENGER DA, BARKER JL: Acetylcholine stimulates cortical precursor cell proliferation in vitro via muscarinic receptor activation and MAP kinase phosphorylation. Eur J Neurosci 12: 1227-1240, 2000.

PALOMEQUE J, RUEDA OV, SAPIA L, VALVERDE CA, SALAS M, PETROFF MV, MATTIAZZI A: Angiotensin II-induced oxidative stress resets the $\mathrm{Ca}^{2+}$ dependence of $\mathrm{Ca}^{2+}$-calmodulin protein kinase II and promotes a death pathway conserved across different species. Circ Res 105: 1204-1212, 2009.

PIROLA CJ, ALVAREZ AL, FINKIELMAN S, NAHMOD VE. Release of acetylcholine from isolated canine renal tissue. Am J Physiol 260: F198-F203. 1991.

ROBEY RB, RUIZ OS, BANIQUED J, MAHMUD D, ESPIRITU DJ, BERNARDO AA, ARRUDA JA: SFKs, Ras, and the classic MAPK pathway couple muscarinic receptor activation to increased $\mathrm{Na}-\mathrm{HCO}_{3}$ cotransport activity in renal epithelial cells. Am J Physiol 280: F844-F850, 2001.

ROSENBLUM K, FUTTER M, JONES M, HULME EC, BLISS TV: ERKI/II regulation by the muscarinic acetylcholine receptors in neurons. $J$ Neurosci 20: 977-985, 2000.

THANGJAM GS, KONDAIAH P: Regulation of oxidative-stress responsive genes by arecoline in human keratinocytes. J Periodontal Res 44: 673-682, 2009.

TSANG MY, RABKIN SW: p38 mitogen-activated protein kinase (MAPK) is activated by noradrenaline and serves a cardioprotective role, whereas adrenaline induces p38 MAPK dephosphorylation. Clin Exp Pharmacol Physiol 36: e12-e19, 2009.

USTUNDAG S, SEN S, YALCIN O, CIFTCI S, DEMIRKAN B, TURE M: L-carnitine ameliorates glycerol-induced myoglobinuric acute renal failure in rats. Ren Fail 31: 124-133, 2009.

VLAHOVIC P, CVETKOVIC T, SAVIC V, STEFANOVIC V: Dietary curcumin does not protect kidney in glycerolinduced acute renal failure. Food Chem Toxicol 45: 1777-1782, 2007.

YOKOZAWA T, OWADA S: Effect of ginsenoside-Rd in cephaloridine-induced renal disorder. Nephron 81: 200-207, 1999.

YOON HJ, MOON ME, PARK HS, KIM HW, IM SY, LEE JH, KIM YH: Effects of chitosan oligosaccharide (COS) on the glycerol-induced acute renal failure in vitro and in vivo. Food Chem Toxicol 46: 710-716, 2008.

ZHANG HA, WANG M, ZHOU J, YAO QY, MA JM, JIANG CL: Protective effect of ginsenoside against acute renal failure and expression of tyrosine hydroxylase in the locus coeruleus. Physiol Res 59: 61-70, 2010. 